\title{
A ATUAÇÃO DO ENFERMEIRO NOS GRUPOS TERAPÊUTICOS DOS CAPS AD DO ESTADO DO ESPÍRITO SANTO
}

Livia Nossa Bourguignon ${ }^{1}$, Élem dos Santos Guimarães², Marluce Miguel de Siqueira ${ }^{3}$

\begin{abstract}
RESUMO: Este estudo teve por objetivo descrever a assistência prestada pelos enfermeiros nos Centros de Atenção Psicossocial Álcool e Drogas-CAPS ad do Espírito Santo, com enfoque nos grupos terapêuticos. Trata-se de um estudo descritivoexploratório, qualitativo, realizado com enfermeiros atuantes em grupos terapêuticos de três CAPS ad, em 2007. Constatou-se nos resultados que a concepção de grupo terapêutico dos enfermeiros depende da experiência vivenciada dentro do próprio grupo e do conhecimento advindo da formação profissional com relação ao tema. A abordagem aplicada e as dificuldades encontradas estão diretamente relacionadas à capacidade de lidar com o processo grupal. Quanto à abordagem teóricometodológica adotada nos grupos terapêuticos, observou-se a utilização do referencial cognitivo comportamental e protocolos do Ministério da Saúde. Este estudo poderá servir de subsídio para repensar o conteúdo aplicado no curso de graduação em Enfermagem referente à temática terapia de grupo, de modo a qualificar a assistência do enfermeiro neste serviço específico. PALAVRAS-CHAVE: Centros de Atenção Psicossocial Álcool e Drogas (CAPS ad); Assistência de enfermagem; Grupo terapêutico.
\end{abstract}

\section{NURSE'S ROLE IN THE 'CAPS AD' THERAPEUTIC GROUPS AT ESPIRITO SANTO STATE}

\begin{abstract}
This study aimed to describe the care provided by nurses in Psychosocial Care Centers Alcohol and Drug (CAPS AD) at Espirito Santo State, specially on therapeutic groups. This is a descriptive exploratory study, qualitative conducted with nurses working in therapeutic groups of three CAPS ad in 2007. It was found in the results that the design of therapeutic group of nurses depends on the lived experience within the group itself and the knowledge gained from training with the issue. The approach applied and the difficulties are directly related to the ability to deal with the group process. As for the theoretical and methodological approach adopted in the therapeutic groups, there was the use of referential cognitive behavioral protocols and the Ministry of Health This study may provide support for rethinking the content applied in the undergraduate program in nursing on the thematic group therapy in order to qualify the assistance of nurses in this particular service.
\end{abstract}

KEYWORDS: Psychosocial Care Centers Alcohol and Drugs (CAPS ad); Nursing care; Group therapy.

\section{LA ACTUACIÓN DEL ENFERMERO EN LOS GRUPOS TERAPÉUTICOS DE LOS CAPS AD DE ESTADO DE ESPÍRITO SANTO}

RESUMEN: Este estudio tuvo por objetivo describir la asistencia prestada por los enfermeros en los Centros de Atención Psicosocial Alcohol y Drogas-CAPS de Espírito Santo, con enfoque en los grupos terapéuticos. Se trata de un estudio descriptivo exploratorio, cualitativo, realizado con enfermeros actuantes en grupos terapéuticos de tres CAPS ad, en 2007. Se ha constatado, en los resultados, que la concepción de grupo terapéutico de los enfermeros depende de la experiencia vivida dentro del propio grupo y del conocimiento advenido de la formación profesional acerca del tema. El abordaje aplicado y las dificultades encuentradas están directamente relacionadas a la capacidad de lidiar con el proceso grupal. Cuanto al abordaje teórico-metodológico adoptado en los grupos terapéuticos, se observó la utilización del referencial cognitivo de comportamiento y protocolos del Ministerio de la Salud. Este estudio podrá servir de subsidio para repensar el contenido aplicado en curso de graduación en Enfermería referente a la temática terapia de grupo, de modo a cualificar la asistencia del enfermero en este servicio específico.

PALABRAS CLAVE: Centros de Atención Psicosocial Alcohol y Drogas (CAPS ad); Asistencia de enfermería; Grupo terapéutico.

\footnotetext{
${ }^{1}$ Acadêmica de Enfermagem e Obstetrícia da Universidade Federal do Espírito Santo-UFES. Bolsista PIBIC/CNPq. Membro da Equipe Técnica do Núcleo de Estudos sobre Álcool e outras Drogas do Centro de Ciências da Saúde-NEAD-CCS-UFES.

${ }^{2}$ Terapeuta Ocupacional da Prefeitura Municipal de Anchieta, Espírito Santo. Mestre em Saúde Coletiva. Membro da Equipe Técnica do NEAD-CCS-UFES.

${ }^{3}$ Enfermeira. Doutora em Enfermagem. Professora Associada II do Departamento de Enfermagem e do Programa de Pós-Graduação em Saúde Coletiva da UFES. Coordenadora Científica do NEAD-CCS-UFES. Orientadora.
}

Autor correspondente:

Lívia Nossa Bourguignon

Universidade Federal do Espírito Santo

Rua Artacerce Broto, 75 - 29065-700 - Vitória-ES, Brasil

Recebido: $16 / 04 / 10$

E-mail: livianossa@hotmail.com

Aprovado: 21/07/10 


\section{INTRODUÇÃO}

Inserindo-se no contexto do processo de reforma psiquiátrica brasileira, os Centros de Atenção Psicossocial (CAPS) assumiram um papel de ressignificação da assistência prestada às pessoas com transtornos mentais. Os CAPS foram criados oficialmente pela portaria GM n. 224, de 29 de janeiro de 1992, e são definidos como

"[...] unidades de saúde locais/regionalizadas que contam com uma população adscrita definida pelo nível local e que oferecem atendimento de cuidados intermediários entre o regime ambulatorial e a internação hospitalar"(1:1168).

Esses serviços foram regulamentados, após atualização, pela portaria GM n. 336, de 19 de fevereiro de 2002, que estabeleceu as diretrizes para funcionamento dos CAPS e as modalidades dos serviços em CAPS I, II, III, i (infanto-juvenil) e ad (álcool e drogas) $)^{(2-3)}$.

Os Centros de Atenção Psicossocial Álcool e Drogas (CAPS ad) representam um grande avanço para a rede de atenção em saúde mental, pois incluem em suas ações serviços, estratégias de promoção, prevenção, tratamento e reabilitação psicossocial, envolvendo todos os níveis de atenção à saúde dos usuários, articulado às demais políticas públicas, constituindo-se um espaço de referência, elemento norteador da rede de serviços substitutivos e porta de entrada para essa população(4).

Das diversas atividades que abordam a temática do álcool e outras drogas nos CAPS ad, o "grupo" se constitui em um dos principais recursos terapêuticos neste contexto de atendimento, sendo regulamentado pelas portarias n. 224/1992 e 336/2002 nas modalidades de: psicoterapia de grupo, grupos operativos, atividades de suporte social e oficinas terapêuticas ${ }^{(2)}$. Entretanto, a temática "grupo" é ainda pouco trabalhada no currículo do curso de Enfermagem, tanto no âmbito da graduação como na pós-graduação, o que corrobora as dificuldades enfrentadas no cotidiano da prática profissional, após uma reforma curricular, na qual a Saúde Coletiva, resultante da saúde mental e saúde pública, deveria representar a lógica do processo de formação do enfermeiro para as intervenções de promoção, prevenção e recuperação da saúde individual e coletiva ${ }^{(5-6)}$.

O trabalho do enfermeiro dentro dos CAPS ad se constitui na reabilitação psicossocial que inclui a reinserção do sujeito nas atividades diárias, no mundo do trabalho e nos espaços comunitários. Este desafio é assumido cotidianamente nas atividades de cuidado, sociais, de acompanhamento, nas oficinas e grupos, enquanto espaços terapêuticos e de socialização ${ }^{(7)}$.

Nos grupos terapêuticos, as finalidades junto à clientela podem ser educativas ou de informação, reflexão e suporte, em que o espaço terapêutico possibilita ao indivíduo sua tomada de consciência como ser social.

Faz-se necessária uma avaliação crítica do modo de intervenção com grupos feita pelo profissional enfermeiro nos CAPS ad, pois o conhecimento que o profissional tem do processo grupal é que irá influenciar neste tipo de intervenção ${ }^{(8)}$.

Para tal, aspectos como o tipo de abordagem empregada nos grupos, bem como a compreensão e preparação dos enfermeiros na prática grupal apontam, assim, a real eficácia desta estratégia terapêutica no âmbito do serviço, para a reinserção e reabilitação de usuários de álcool e outras drogas. Nesse sentido, o objetivo deste estudo foi descrever o trabalho do enfermeiro nos CAPS ad, com enfoque nos grupos terapêuticos que esse profissional orienta.

\section{METODOLOGIA}

A pesquisa de caráter descritivo-exploratória e abordagem qualitativa foi realizada no período de junho a agosto de 2007, com enfermeiros dos CAPS ad do Estado do Espírito Santo, localizados nos Municípios de Vila Velha, Serra e Vitória, únicos serviços nessa modalidade de atenção existentes até a data da coleta dos dados. O CAPS ad de Vitória, mais conhecido como Centro de Prevenção e Tratamento de Toxicômanos (CPTT), foi o primeiro serviço a ser implantado no Estado para o atendimento de usuários de álcool e outras drogas, iniciando suas atividades em 1993 e tornando-se um serviço de referência para todo o Estado. Em 2003 e 2006, como forma de ampliar a rede de atenção psicossocial na atenção aos usuários de álcool e outras drogas, foram implantados mais dois serviços na modalidade CAPS ad, nos Municípios de Vila Velha e Serra, respectivamente.

A pesquisa de campo buscou realizar a descrição e exploração dos grupos terapêuticos realizados em cenários específicos e teve como objetivo descrever a assistência prestada pelo enfermeiro nesses grupos. Os sujeitos deste estudo foram três enfermeiros que coordenavam ou atuavam nos grupos terapêuticos 
dos CAPS ad. Os sujeitos aceitaram participar da coleta de dados, assinando o Termo de Consentimento Livre e Esclarecido, após a elucidação do projeto, conforme Resolução n. 196/96 do Conselho Nacional de Saúde. O projeto foi aprovado pelo Comitê de Ética em Pesquisa (CEP) da UFES, pelo processo n. 055/09. A coleta dos dados foi feita através de entrevistas com um roteiro semiestruturado, pela pesquisadora, no próprio local de trabalho dos sujeitos e durante a jornada de trabalho. Os dados foram tratados segundo a análise de conteúdo ${ }^{(9)}$ e agrupados em categorias, a saber: 1) Modo de atuação do enfermeiro nos grupos terapêuticos junto a usuários de álcool e outras drogas; 2) Limites e possibilidades para realização destes grupos aos usuários.

A análise possibilitou compreender os significados no contexto da fala dos sujeitos, o que constituiu o corpus da pesquisa. Nesta perspectiva, a análise da frequência das falas e palavras ou unidades de significação permitiram o alcance do núcleo de compreensão dos textos e uma interpretação mais profunda ${ }^{(10)}$.

\section{RESULTADOS E DISCUSSÃO}

\section{Caracterização dos Grupos Terapêuticos}

No tocante às atividades grupais, observou-se uma diversidade de ofertas terapêuticas e denominações diferenciadas nos grupos desenvolvidos nos CAPS ad, sendo chamados de grupos de acolhimento, acompanhamento, oficinas terapêuticas e assembleias.

Os Grupos de Acolhimento são considerados a porta de entrada do serviço. São grupos voltados para recepção dos usuários que buscam o serviço pela primeira vez, por demanda própria ou por encaminhamento dos serviços de saúde, da Justiça ou por outros serviços. Nesses grupos, apresenta-se o funcionamento do serviço, colhe-se a história de vida, traça-se o projeto terapêutico e fazem-se os encaminhamentos necessários, permitindo que o usuário também expresse suas expectativas e demandas. Os Grupos de Acolhimento são realizados por dois profissionais da equipe técnica e acontecem até três vezes por semana e atendem cerca de 15 pessoas, dependendo da demanda do serviço. São grupos voltados para o diálogo e escuta e têm por objetivo fazer uma anamnese geral dos usuários que chegam ao serviço, para posteriormente proceder aos encaminhamentos necessários.

Os Grupos de Acompanhamento são considerados grupos terapêuticos mais específicos voltados para clientelas e demandas específicas. Acontecem, geralmente, uma vez por semana e atendem até 15 pessoas. São grupos classificados segundo a faixa etária (grupo de adolescentes, grupo de 18 a 25 anos, grupo acima de 45 anos), gênero (grupo de mulheres, grupo de homens) e, em alguns serviços, são classificados segundo a utilização de um tipo de droga (grupo de álcool, grupo de múltiplas drogas). Nesses grupos, várias estratégias são utilizadas, abordando temas diversos; é um grupo de discussão e reflexão, que se utiliza do diálogo, de dinâmicas e técnicas para gerar algum tipo de discussão.

As Oficinas são uma das principais formas de tratamento oferecidas nos CAPS ad. São atividades da atenção diária, voltadas para usuários que necessitam de uma atenção mais intensiva. São consideradas atividades grupais de socialização, expressão e inserção social. Diferenciam-se dos Grupos de Acolhimento e Acompanhamento pela frequência das atividades que são mais intensas. As Oficinas podem ter um caráter produtivo (possibilitando o aprendizado de atividades que podem servir para geração de renda), educativo (aprendizagem de alguma técnica ou habilidade), terapêutico (espaço de reflexão, expressão corporal, expressão artística, transformação e criação) informativo ou expositivo ${ }^{(2)}$.

De modo geral, nota-se que o atendimento prestado no âmbito das atividades grupais se dá de diversas maneiras, dependendo da modalidade de atenção (intensiva, semi-intensiva ou não-intensiva) demandada pelo usuário do serviço.

\section{Caracterização do Modo de Atuação e dos Desafios para o Enfermeiro}

\section{Modo de atuação do enfermeiro nos grupos terapêuticos}

Para a realização dos grupos terapêuticos, o enfermeiro recorre a conhecimentos provenientes da graduação, materiais pesquisados de diversas fontes metodológicas e manuais do Ministério da Saúde parase atualizar quanto à dinâmica de grupo promovendo, assim, a assistência preconizada nas portarias de criação e regulamentação dos CAPS.

\section{Conceito de grupo do enfermeiro}

Os significados dados pelos enfermeiros ao conceito de grupo apontaram semelhanças, como a que 
relata o grupo como um conjunto de algumas pessoas reunidas com uma determinada finalidade em comum:

[...] eu acho que grupo é quando tem mais de uma pessoa juntas. (E.1)

São algumas pessoas, que se reúnem com a mesma problemática, pra discutir certos assuntos, problemas que eles estão enfrentando [...]. (E.2)

É um conjunto de pessoas que estão unidas com uma determinada finalidade. Tem que ter uma finalidade em comum para unir todos para formar um grupo. (E.3)

Estes significados apresentados pelos enfermeiros se aproximam muito das características básicas de um grupo, descritas por diversos autores. Um grupo se caracteriza por todos que o integram estarem reunidos em tarefa e objetivo comuns ${ }^{(11)}$. O grupo pode ser um conjunto de pessoas que estão ligadas por interesses em comum, numa ação interativa e compartilhada; mas, para que se configure verdadeiramente um grupo, é necessário que haja alguma interação entre os indivíduos ${ }^{(12)}$. Também, o grupo é conceituado como um conjunto de pessoas ligadas pelo tempo e espaço as quais se propõem à realização de uma tarefa ou uma finalidade ${ }^{(13)}$.

Todos estes autores corroboram o caso específico nos CAPS ad, em que a ligação pela problemática do álcool e outras drogas entre os usuários deste serviço e a superação pressupõe o objetivo ou a tarefa em comum.

\section{Abordagem teórico-metodológica utilizada nos grupos pelo enfermeiro}

Em relação à abordagem teórico-metodológica aplicada, os enfermeiros fizeram referência à abordagem cognitivo-comportamental e aos protocolos do Ministério da Saúde:

Eu gosto muito da abordagem cognitiva comportamental, é a experiência que eu tenho no Grupo de Tabagismo. Creio assim, a abordagem cognitiva comportamental ela é simples de ser aplicada é é bem diretiva [...]. (E.2)

Eu me baseio no Ministério da Saúde. Eu pego o protocolo do Ministério e utilizo cada tema que, geralmente, eles [usuários] escolhem o tema que eles querem ter a palestra de saúde, que é uma vez por mês. (E.3)

A Terapia Cognitivo-Comportamental está centrada nos problemas e questões trazidos pelo paciente, objetivando ajudá-lo a aprender novas estratégias para atuar no ambiente, de forma a promover mudanças necessárias. A metodologia utilizada na terapia é de uma cooperação entre o terapeuta e o paciente de forma que as estratégias para a superação de problemas concretos são planejadas em conjunto ${ }^{(14)}$.

Os protocolos e diretrizes do Ministério da Saúde visam a sistematização de medidas técnicas e operacionais, em que a fundamentação para o desenvolvimento das ações assistenciais do serviço contem elementos inerentes às políticas, sendo direcionadas aos usuários de álcool e outras drogas. É importante que o profissional que deseja trabalhar com grupos tenha clareza quanto aos seus objetivos e métodos. É preciso considerar os aspectos estruturais e do desenvolvimento do grupo, no estabelecimento de uma abordagem para não ficar sujeito a erros ou ao insucesso na realização da tarefa. Isso poderá acontecer devido ao desenvolvimento informal dos grupos terapêuticos, utilizando abordagens sem fins terapêuticos, que trabalhem de forma sistematizada visando tais objetivos. Há alguns requisitos básicos a serem considerados na caracterização dos grupos, sejam eles operativos ou com objetivos terapêuticos, tais como: sua estruturação, finalidade para qual eles foram criados, a dinâmica grupal, o procedimento técnico empregado ou o referencial teórico adotado.

\section{Limites e possibilidades para realização destes grupos aos usuários}

\section{Dificuldades nos grupos terapêuticos}

Entre as dificuldades citadas pelos profissionais enfermeiros para realizar os grupos terapêuticos nos CAPS ad está a escassez de material para realização de dinâmicas, trabalhos manuais, confecção de cartazes, visualização de vídeos e filmes, levando-os a fazer um investimento pessoal no serviço, conforme aponta a fala abaixo:

[...] por exemplo, quero fazer a dinâmica da carametade, eu vou fazer tudo do meu bolso. Vou comprar a cartolina, vou fazer em casa, não vou deixar de fazer até para motivar que eles continuem participando. 
Material que temos é o que a gente traz de fora. (E.1)

O trabalho em si não se restringe à técnica. Elementos como subjetividade (mobilização, investimento pessoal, singularidade, cognição, imaginação, criatividade) e que este seja permeado pelo social (por relações de cooperação e compreensão) - que relata a geração de sofrimento ao trabalhador quanto à tentativa de execução do trabalho - neste sentido mais amplo, não são reconhecido ${ }^{(15)}$.

Outra dificuldade apontada foi o espaço físico que o serviço oferece:

[...] bom, outra dificuldade é acomodar todo mundo numa sala só, porque aqui não tem como. O espaço físico é precário [...]. (E.3)

A estrutura grupal básica baseia-se em pressupostos que garantem o seu bom funcionamento, dentre os quais insere-se o enquadre (setting) do grupo: as condições físicas do ambiente para a realização das atividades são fundamentais, tais como uma sala ampla, arejada, iluminada, com acomodação para todas as pessoas; um setting circular das cadeiras é recomendado, pois facilita a visualização dos participantes entre si. O coordenador deve cuidar, na escolha do local, para que não ocorram interrupções consideradas indesejadas. Seu tamanho não pode exceder o limite que ponha em risco a indispensável preservação da comunicação, tanto a visual como a auditiva, e a conceitual. Deve-se estabelecer o setting e o cumprimento dos combinados feitos no grupo (contrato terapêutico). Portanto, o grupo deve ter objetivos claros e contar com uma estabilidade de espaço (local das reuniões), de tempo (horários) e com algumas regras que delimitam e normalizam a atividade grupal proposta ${ }^{(16)}$.

O grau de estrutura dependerá do tipo de funcionamento e organização interna do grupo, e cabe ao coordenador explicitá-los na fase de planejamento do trabalho ${ }^{(5)}$. Os tipos de grupos podem ser caracterizados através da descrição de seu funcionamento. De modo geral, constatou-se que os cenários investigados não contavam com condições adequadas para a realização das atividades de grupo, como descritas nas diretrizes de estruturação dos CAPS ad. Verificou-se que a maioria dos CAPS ad do Espírito Santo não estão estruturados com as condições mínimas conforme as normalizações da Política de Saúde Mental do Estado. Este problema se estende à rede de
Saúde Mental do Estado, que se apresenta em fase de implementação ${ }^{(17)}$.

A complexidade da demanda por a adictos de álcool e drogas consiste em uma dificuldade que impõe muitos desafios aos profissionais, uma vez que a composição de um grupo engloba os diferentes gêneros, níveis sociais, econômicos, ou seja, diferentes realidades que implicam na heterogeneidade deste grupo, o que requer do coordenador sensibilidade para lidar com essas questões. Um grupo homogêneo é formado por pessoas que apresentam uma série de fatores e de características comuns a todos os membros. Já nos grupos heterogêneos, há uma composição variada em sexo, idade, doença, nível socioeconômico, cultura etc. A composição do grupo foi pontuada por um dos enfermeiros:

[...] como é um grupo que é unido pela questão de drogas, a gente tem assim pessoas de várias classes sociais, vários niveis de instrução. Então alguns não conseguem entender muito bem o que é dito no grupo [...]. (E.3)

Segundo a pesquisa, os grupos realizados nos CAPS ad apresentavam-se homogêneos quanto à problemática (usuário de álcool e outras drogas) e heterogêneos quanto aos aspectos do nível sociocultural. O nível de interação em um grupo muito homogêneo pode se dar superficialmente, não gerando grandes discussões e reflexões. Em contrapartida, um grupo muito heterogêneo pode desencadear desentrosamento entre os participantes, bem como dificuldades e resistência ao coordenador. Cabe, portanto, ao coordenador estar preparado e saber contornar as dificuldades que estes grupos possam apresentar, garantindo assim que, independentemente da composição que estes possuam - se conduzidos e explorados de fato - o objetivo possa ser alcançado.

Outra dificuldade apontada foi o não gostar de realizar grupos, como observada na fala:

Tenho dificuldade com pessoas, por não gostar de trabalhar com grupos [...].

Um fator de fundamental importância na realização do grupo é o seu coordenador, sendo aqui empregado no sentido mais amplo das diferentes profissões e tipos de grupo. Este coordenador é peçachave na evolução do grupo, sendo necessário que possua atributos que o levem a assumir a condição que 
este trabalho impõe, de liderança e de responsabilidade, de acordo com os valores e características que um grupo adquire. Além dos conhecimentos provindos de muito estudo e leituras e de habilidades (treino e supervisão), as atitudes (um tratamento de base psicanalítica ajuda muito) são indispensáveis, bem como gostar e acreditar em grupos, ser continente, ser empático, comunicativo, verdadeiro, ter senso de humor, ter capacidade de integração e síntese, de discriminação e identificação ${ }^{(18)}$.

\section{Preparo técnico do enfermeiro para fazer grupos}

A formação acadêmica revelou-se um determinante para a aquisição de conhecimentos teóricos e práticos específicos sobre álcool e outras drogas, bem como ser um divisor de águas quando o profissional necessita aplicar tais conhecimentos.

[...] a minha formação foi bem curativa, pra trabalhar em hospital. Na minha época, a Saúde Pública era muito fraquinha. Éramos bem preparados para trabalhar em hospital, não tinha outro caminho [...]. (E.1)

[...] o que eu aprendi foi mais didático, educação em saúde, e [o trabalho] é mais na prática mesmo. (E.3)

Quanto à preparação do terapeuta para coordenação de grupo, o profissional iniciante, pela própria inexperiência, pode sentir-se ansioso, com tendência a ser defensivo e resistente. Estes mecanismos podem levar à evasão ou término de um grupo. É sugerida, como técnica de formação do profissional, a surpevisão por profissional específico, pois esta oferece ao supervisionado a oportunidade de examinar com mais detalhes o que está fazendo enquanto terapeuta. É importante ressaltar que é preciso investigar em que medida os profissionais tiveram ou têm contato com ações de capacitação/formação para lidar com demandas que o atendimento requer, pois isso irá influenciar nas suas práticas assistenciais ${ }^{(8)}$.

Refletindo sobre o ensino da temática "grupos" na Graduação em Enfermagem, o currículo atual não apresenta uma uniformidade neste aspecto. É evidente que o enfermeiro não possui preparo teóricometodológico para explorar o grupo como referencial e que poucos conteúdos que abordam o ensino da coordenação de grupos ou equipes; e, se abordados, encontram-se vinculados a diversas disciplinas, não tendo enfoque terapêutico específico. Pode-se afirmar que o tema ainda é pouco abordado nas instituições de ensino superior de todo país e, embora a temática seja de extrema relevância, ainda é incipiente o número de cursos que tratam o conteúdo na formação do enfermeiro ${ }^{(19)}$.

\section{CONSIDERAÇÕES FINAIS}

Para que as possibilidades terapêuticas aumentem por meio do processo terapêutico grupal de atenção à saúde, é necessário que o profissional esteja preparado para reconhecer e compreender a crise motivadora da procura da ajuda. Para isso, é necessário que sua assistência promova informação, educação em saúde, reflexão e suporte. O grupo é uma forma de explorar estes aspectos, e deve perpassar a necessidade de atender uma grande demanda ou uma forma informal de prestar assistência.

O profissional enfermeiro deve se apropriar da ferramenta "grupos terapêuticos" como suporte tecnológico para a Enfermagem, uma vez que estes constituem, atualmente, a principal estratégia terapêutica empregada nos CAPS ad. A formação do enfermeiro deve, portanto, contribuir não só para a visão dinâmica dos serviços de saúde, onde o enfermeiro se insere, mas para a coordenação de grupos e equipes de saúde.

Para o aprendizado do processo grupal e do seu funcionamento, não basta o estudo teórico, faz-se necessário o cotidiano da prática. Embora a temática seja de extrema relevância, ainda é incipiente o tratamento do conteúdo na formação do enfermeiro, que, embora preconize o cuidado do indivíduo em uma visão holística, ainda se constitui em um modelo centrado no doente e na doença. Sugere-se, portanto, a busca de métodos e estratégias de ensino que possam impactar na mudança do estilo de vida e, consequentemente, melhoria na assistência à saúde.

Sugere-se novos estudos acerca do tema, procurando ouvir os usuários dos CAPS ad, ou seja, os verdadeiros atores dos grupos terapêuticos, de forma a se apreender e se aprender fazendo. É estratégico conhecer qual a percepção destes nas relações estabelecidas, nos métodos aplicados e conhecer os resultados desse processo grupal como estratégia terapêutica.

\section{REFERÊNCIAS}

1. Brasil. Portaria/SAS n. 224, de 29 de Janeiro de 1992. Normatiza o atendimento em saúde mental na rede 
SUS. Diário Oficial da República Federativa do Brasil. Brasília, 30 jan. 1992. Seção 1:1168.

2 Brasil. Portaria/GM n. 336, de 19 de fevereiro de 2002. Define e estabelece diretrizes para o funcionamento dos Centros de Atenção Psicossocial. Diário Oficial da República Federativa do Brasil. Brasília, 17 set. 2004. Seção 1:51.

3. Mello MF, Mello AAF, Kohn R, organizadores. Epidemiologia da saúde mental no Brasil. Porto Alegre: Artmed; 2007.

4. Siqueira MM, Barbosa DA, Laranjeira R. As políticas públicas relacionadas às substâncias psicoativas. Enfermagem Atual. 2008;45:25-9.

5. Munari DB, Rodrigues ARF. Enfermagem e grupos. Goiânia: AB; 1997.

6. Kantorski LP, Mielke FB, Teixeira Júnior S. O trabalho do enfermeiro nos centros de atenção psicossocial. Trab Educ Saúde. 2008;6(1):87-105.

7. Mafacciolli R. Os grupos na atenção básica de saúde de Porto Alegre: uso e modos de intervenção terapêutica [dissertação]. Porto Alegre (RS): Universidade Federal do Rio Grande do Sul; 2006.

8. Bardin L. Análise de conteúdo. Lisboa: Edições 70; 1979.

9. Minayo, MCS. O desafio do conhecimento: pesquisa qualitativa em saúde. São Paulo: HUCITEC; 2008.

10. Zimerman DE. Fundamentos básicos das grupoterapias. $2^{\mathrm{a}}$ ed. Porto Alegre: Artes Médicas; 2000.

11. Osório LC. Grupos: teorias e práticas - acessando a era da grupalidade. Porto Alegre: Artes Médicas; 2000.

12. Pichón-Riviére E. O processo grupal. $7^{\mathrm{a}}$ ed. São Paulo: Martins Fontes; 2005.

13. Lima MVD, Wielenska RC. Terapia comportamentalcognitiva. In: Cordioli AV, organizador. Psicoterapias: abordagens atuais. Porto Alegre: Artes Médicas; 1993. p. 192-209.

14. Oliveira NT. Somatização e sofrimento no trabalho. Texto \& Contexto Enferm. 2003;2(1):1-14.

15. Vinogradov S, Yalom ID. Manual de psicoterapia de grupo. Porto Alegre: Artes Médicas; 1992.

16. Santos EG. O grupo como estratégia terapêutica nos Centros de Atenção Psicossocial Álcool e Drogas do Espírito Santo [dissertação]. Vitória (ES): Universidade Federal do Espírito Santo; 2010.

17. Zimerman DE, Osório LC. Como trabalhamos com grupos. Porto Alegre: Artes Médicas; 1997.

18. Pinto AGA, Jorge MSB. Prática clínica em saúde mental no cotidiano do centro de atenção psicossocial. Cogitare Enferm. 2009;14(2):217-26.

19. Munari DB, Rocha BS, Nunes DS, Medeiros M. O ensino da temática de grupo nos cursos de graduação em enfermagem no Brasil. Rev Gaúcha Enferm. 2005;26(2): 220-30. 